\title{
Electronic properties of dislocations
}

\author{
M. Reiche ${ }^{1} \cdot$ M. Kittler ${ }^{2}$ H. Uebensee ${ }^{3} \cdot$ E. Pippel $^{1} \cdot$ A. Haehnel $^{1}$. \\ S. Birner ${ }^{4}$
}

Received: 8 June 2015 / Accepted: 8 November 2015/Published online: 14 March 2016

(c) The Author(s) 2016. This article is published with open access at Springerlink.com

\begin{abstract}
Dislocations exhibit a number of exceptional electronic properties resulting in a significant increase in the drain current of MOSFETs if defined numbers of these defects are placed in the channel. Measurements on individual dislocations in $\mathrm{Si}$ refer to a supermetallic conductivity. A model of the electronic structure of dislocations is proposed based on experimental measurements and tightbinding simulations. It is shown that the high strain level on the dislocation core-exceeding $10 \%$ or more-causes locally dramatic changes in the band structure and results in the formation of a quantum well along the dislocation line. This explains experimental findings (two-dimensional electron gas, single-electron transitions). The energy quantization within the quantum well is most important for supermetallic conductivity.
\end{abstract}

\section{Introduction}

Dislocations are one-dimensional crystal defects influencing many of the physical and mechanical properties of crystalline solids [1, 2]. The analysis of their electronic properties is of particular interest for semiconductor devices because dislocations detrimentally effect

\section{Reiche}

reiche@mpi-halle.de

1 Max Planck Institute of Microstructure Physics, Weinberg 2, 06120 Halle/S., Germany

2 Brandenburg University of Technology Cottbus-Senftenberg, Platz der Deutschen Einheit 1, 03046 Cottbus, Germany

3 CIS Research Institute of Microsensorics and Photovoltaics, K.-Zuse-Str. 14, 99099 Erfurt, Germany

4 nextnano GmbH, Südmährenstr. 21, 85586 Poing, Germany device parameters. This was proved, for instance, by electron-beam-induced current (EBIC) method [2, 3]. Detailed investigations by various experimental techniques [Hall effect, electron paramagnetic resonance (EPR), deep-level transient spectroscopy (DLTS), photoluminescence] revealed numerous deep and shallow defect levels in the gap of $\mathrm{Si}$ related to dislocations [48]. Because most of the investigations in the past were carried out on deformed silicon, it is not clear which of the defect levels are related to the dislocation core, or to interactions with point defects in the strain field surrounding a dislocation [6]. Measurements on two-dimensional dislocation networks produced by wafer direct bonding avoid these interactions. Recently published DLTS data refer also to different deep and shallow levels in $\mathrm{p}$ - and n-type $\mathrm{Si}[9,10]$. Their existence depends on the dislocation density. Shallow levels having activation energies of about $0.1 \mathrm{eV}$ are discussed as possible reasons of radiative recombination (D1 luminescence) [11], while an interpretation of deep levels, related to the dislocation core, is still missing. Depending on defect formation, the energies of deep levels spread over a large energy range in the gap [6].

Dislocations also cause a significant increase in the drain current $\left(I_{\mathrm{D}}\right)$ of metal-oxide-semiconductor field-effect transistors (MOSFETs) if well-defined numbers of parallel dislocations are placed in the channel [12]. The largest increase in $I_{\mathrm{D}}$ is obtained for a single dislocation [13]. In p-type silicon dislocations form channels for electrons with supermetallic conductivity which is about eight orders of magnitude higher than of the surrounding Si matrix [13]. This exceptional property and their dimension (length of a few micrometers and a cross-sectional area of the defect core of about $1 \mathrm{~nm}^{2}$ ) characterize dislocations as native nanowires embedded in a perfect crystalline matrix. 
In a previous paper, we expect that a reason of the supermetallic behavior is the high strain $\varepsilon$ in the dislocation core, which exceeds $\varepsilon \cong 10 \%$ or more resulting in local modifications of the band structure and carrier mobility [13]. The present paper presents an experimental and theoretical description of the electronic properties of dislocations based on the strain in the dislocation core. This allows us to explain the supermetallic behavior as well as deep levels proved by DLTS. Selected results of experimental measurements of electrical properties of dislocations and of local strain fields present Chapter 3. Computer simulations of the band structure using tight-binding and k.p models are discussed in Chapter 4. A model of the electronic structure of a dislocation is proposed in Chapter 5.

\section{Experimental}

The technique to realize defined two-dimensional arrays of dislocations in thin layers (semiconductor wafer direct bonding) was described elsewhere [13, 14]. Silicon-on-insulator (SOI) wafers (diameter $150 \mathrm{~nm}$, buried oxide (BOX) thickness $60 \mathrm{~nm}$, device layer thickness $30 \mathrm{~nm}$ ) were applied for the wafer bonding process under hydrophobic conditions. One of the handle wafers was removed after bonding resulting in a new SOI wafer having a device layer thickness of $60 \mathrm{~nm}$ and a two-dimensional dislocation network in the middle. SOI MOSFETs and pndiodes were prepared on such substrates (for details see [14]). Electrical measurements of device parameters were carried out in the temperature range from 300 to $3 \mathrm{~K}$.

The defect structure was analyzed by applying a probe $C_{\mathrm{S}}$ corrected scanning transmission electron microscope (FEI Titan 80-300). Local strain fields are quantified by peak-pairs analysis (PPA) of high-resolution electron microscope images.

All simulations of the band structure were performed by application of the nextnano ${ }^{3}$ simulation package [15]. The bulk band structure was calculated using both a 6- and 8 -band k·p model, as well as an empirical $s p^{3} d^{5} s^{*}$ tightbinding model [16] taking into account strain and deformation potentials.

\section{Experimental evaluation}

\subsection{Electrical measurements}

Well-defined sets of parallel dislocations in the channel of nMOSFETs result in a dramatic increase in the drain current $\left(I_{\mathrm{DS}}\right)$. Figure 1 shows the transfer characteristics of devices without and with dislocations. The MOSFET

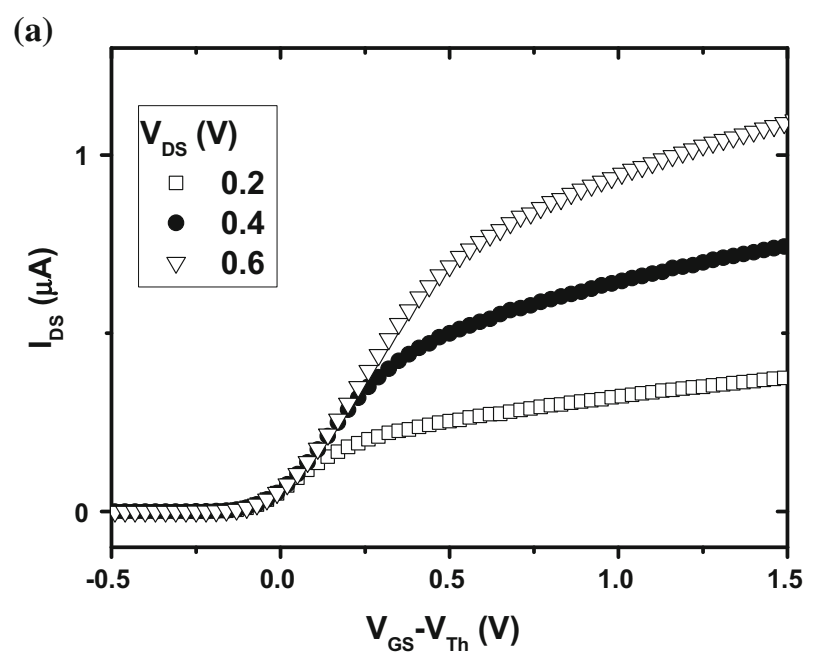

(b)

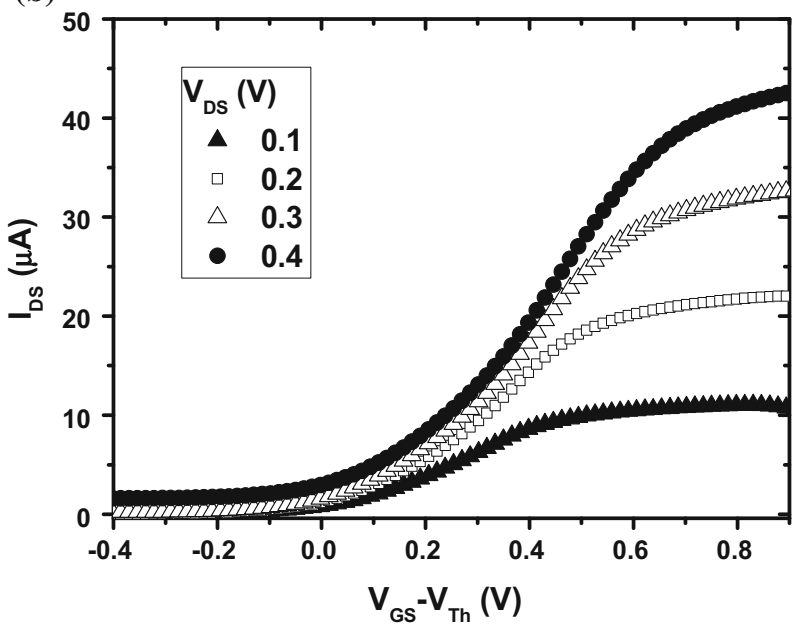

Fig. 1 Transfer characteristics $\left(I_{\mathrm{DS}}-V_{\mathrm{GS}}\right)$ of nMOSFETs without (a) and with dislocations in the channel (b). The gate voltage $V_{\mathrm{GS}}$ is represented as difference $V_{\mathrm{GS}}-V_{\mathrm{Th}}$ with $V_{\mathrm{Th}}$ as threshold voltage. Note the different scale

without dislocations (reference) was fabricated on an identic SOI wafer without a dislocation network in the 60 -nm-thick device layer and under the same process conditions. At $V_{\mathrm{GS}}=0.8 \mathrm{~V}$ and drain voltage $\left(V_{\mathrm{DS}}\right)$ of $0.4 \mathrm{~V}$, the drain current is about $40 \mu \mathrm{A}$ for MOSFETs containing dislocations in the channel while in the same time $I_{\mathrm{DS}}$ is only $0.6 \mu \mathrm{A}$ for a reference device. This corresponds to a current increase by a factor of 67 . The increase in $I_{\mathrm{DS}}$ depends on the type and the number of dislocations in the channel. The largest increase in $I_{\mathrm{DS}}$ is observed if mixed dislocations are present.

The increase in the drain current for nMOSFETs results from an increased electron transport along dislocations. On the other hand, an increase in $I_{\mathrm{DS}}$ exists also for pMOSFETs where holes are involved in the carrier transport along dislocations. This means that depending on the 


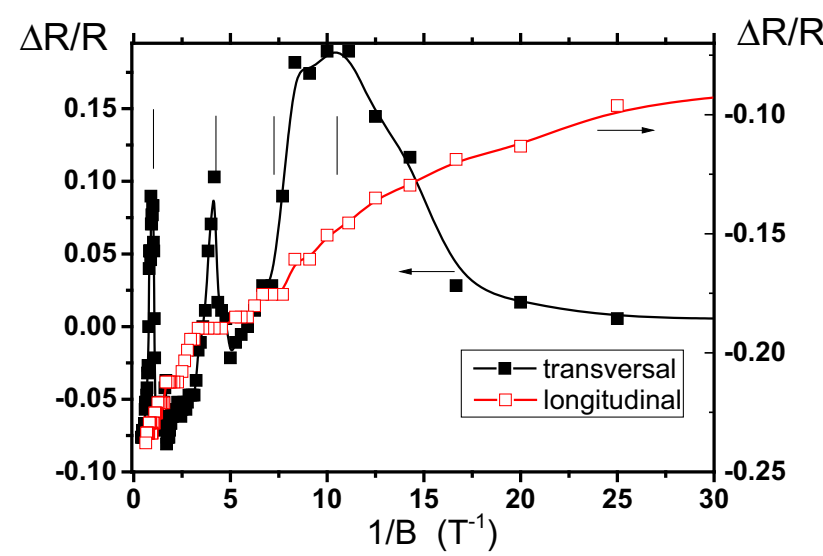

Fig. 2 Transversal and longitudinal source-drain resistivity $(\Delta R / R)$ as a function of the inverse magnetic field $B$. Measurement on an nMOSFET with a dislocation network $\left(V_{\mathrm{DS}}=3 \mathrm{~V}\right)$ at $3.1 \mathrm{~K}$

properties of the surrounding material (p- or n-type), dislocations transport either electrons or holes. The dislocation core is occupied by electrons in p-type material and by holes in n-type material. This is in contrast to the basic model of electronic states on dislocations [4] assuming holes on the core of a dislocation in p-type material surrounded by the so-called Read cylinder of ionized (negatively charged) acceptor atoms.

Indications of a reinterpretation of the dislocation model provide also low-temperature measurements. Magnetoresistance measurements revealed Shubnikov-de Haas $(\mathrm{SdH})$ oscillations referring to the presence of a two-dimensional electron gas (2DEG) on dislocations networks in p-type silicon (Fig. 2). The asymmetry of the $\mathrm{SdH}$ oscillations is frequently observed, but different reasons are discussed (for instance [17, 18]). Because dislocations represent nanowires, the asymmetry may be attributed to the fact that field-dependent oscillations are extremely sensitive to the alignment between the field and the wire axis [19]. As a consequence of the 2DEG, single-electron transitions (Coulomb blockades) are observed [20]. Coulomb blockades are also found in dislocated n-type Si referring to the confinement of holes.

\subsection{Electron microscopy}

If $\{100\}$-oriented surfaces of $\mathrm{Si}$ are bonded together, a network of screw dislocations is formed in the bonded interface by the twist misorientation. The network is formed by two sets of screw dislocations with Burgers vectors $\mathbf{b}=1 / 2<110>$ oriented parallel to $<110>$ directions. Depending on the annealing conditions subsequently followed after the initial bonding, screw dislocations mostly dissociate into Shockley partials according to reactions of the type
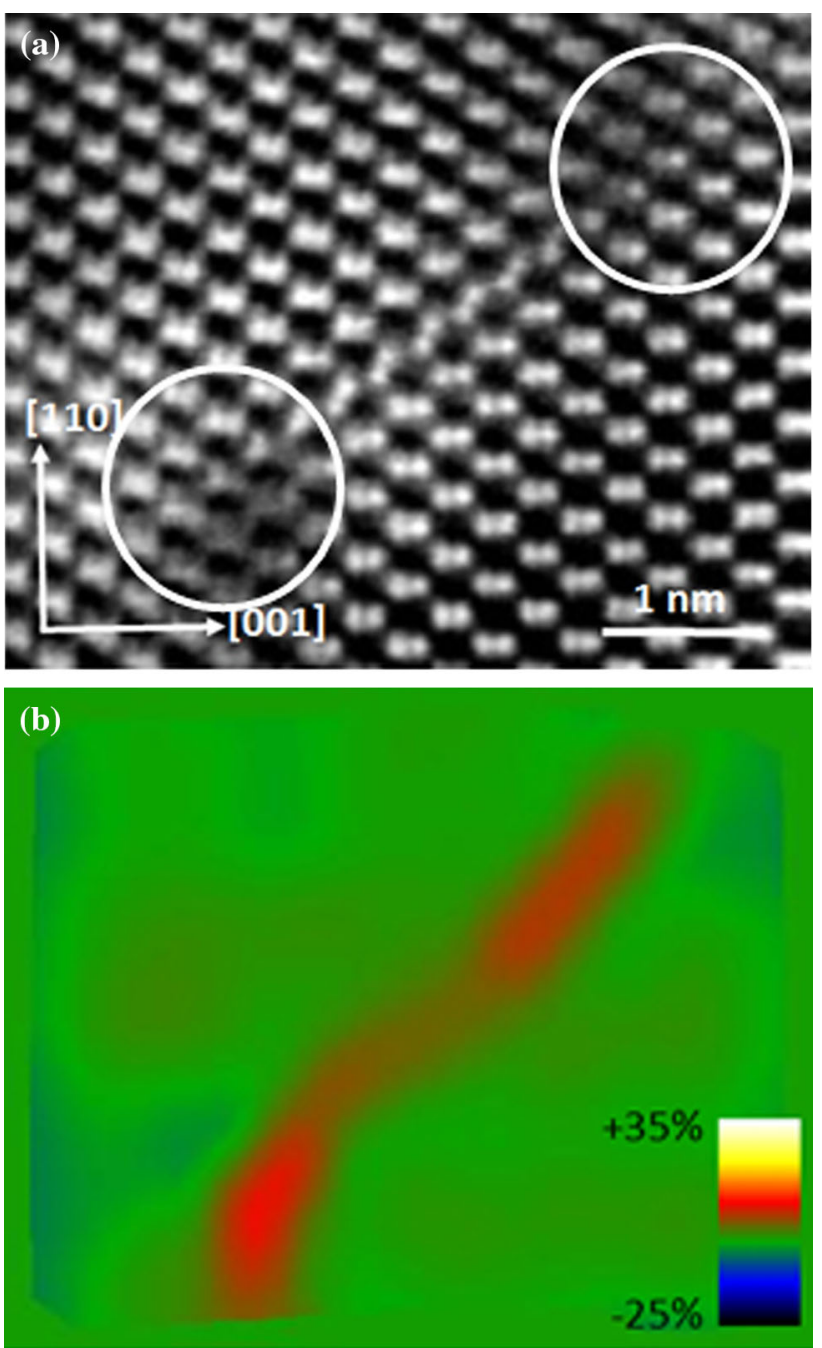

Fig. 3 HAADF image of a dissociated screw dislocation (a). A $\{110\}$ cross-section sample was used for imaging. The resulting inplane strain $\left(\varepsilon_{x y}\right)$ in $\{110\}$ is tensile $\left(\varepsilon_{x y} \cong 10 \%\right)$ and concentrated on the partial dislocations (b)

$\frac{1}{2}[110]=\frac{1}{6}[12 \overline{1}]+\frac{1}{6}[211]$

Both partial dislocations are oriented parallel to $<110>$ directions in the $\{111\}$ glide plane. Their Burgers vectors are therefore inclined at an angle of $30^{\circ}$ to the dislocation line direction. In addition, the dissociation reaction results in a stacking fault between both partial dislocations. Figure 3a shows a high-angle annular dark field (HAADF) microscopy image of a dissociated screw dislocation. The positions of the partial dislocations are indicated in the figure by circles. The strain field at the dislocation core and around the defect is quantified by peak-pairs analysis (PPA) [21]. PPA extracts the strain $\varepsilon$ by measurement of the lattice distortion. Analyses of $\varepsilon$ clearly proved that most of the strain is located on the dislocation core (Fig. 3b). 
Inside the defect, tensile strain of about $10 \%$ exists, which is in accordance with theoretical predictions [22, 23] and measurements on other defects [24]. The measured strain in the dislocation core is much higher than in the surrounding strain field. Furthermore, misfit of the tilt component of both bonded wafers results in an additional network of socalled $60^{\circ}$ dislocations representing edge dislocations in the diamond cubic structure [25]. $60^{\circ}$ dislocations dissociate also, but in $30^{\circ}$ and $90^{\circ}$ partials. The latter have Burgers vectors perpendicular to the dislocation line introducing normal strain, which is compressive above and tensile below the slip plane.

\section{Band structure}

The effect of different types of strain (uniaxial, biaxial, tensile, and compressive) on the band structure of $\mathrm{Si}$ and device properties has been analyzed using different simulation techniques [26-31]. The principal structure for our investigations is an nMOSFET schematically shown in Fig. 4. A dislocation is placed in the middle of the channel parallel to $<110>$. This introduces uniaxial strain parallel to the channel direction. Band structure calculations revealed shifts of the conduction and valence bands at the $\Gamma$ - and $L$-point as well as band warping with increasing tensile strain. The conduction-band minimum at $\Delta$ is nearly constant with increasing tensile strain. If $\varepsilon=+10 \%$, the lowest conduction band at the $\Gamma$ point $\left(\Delta E_{c}^{\Gamma}\right)$ is equal to the position of the lowest conduction band at $\Delta$ of the unstrained Si. As a result, the band gap energies of the indirect and direct transitions are equal for such high tensile strain and $\mathrm{Si}$ changes from the indirect into a direct semiconductor. This conclusion is in agreement with previously reported data [30, 31]. Furthermore, the shift of the conduction-band minimum at the $L$-point results in an additional indirect transition in the presence of high tensile strain. Using the model of a dislocation surrounded by unstrained silicon (channel, Fig. 4), a schema of the band

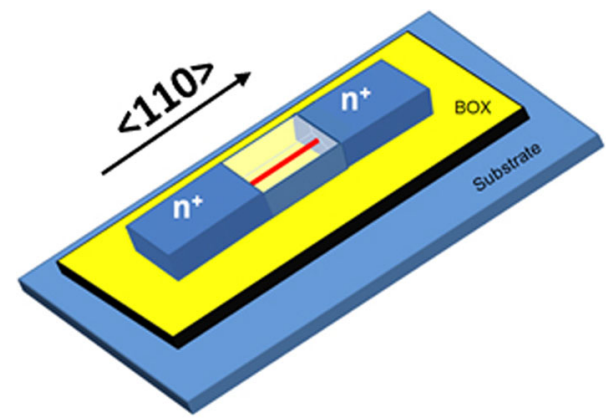

Fig. 4 Schema of nMOSFET with a dislocation (red line) in the channel. The overlying gate is not shown for clearness structure as in Fig. 5 follows. The shifts of the conduction band of the strained dislocation core result in one or more one-dimensional defect bands in the band gap of the surrounding $\mathrm{Si}$ channel. The depth of the defect bands increases as the tensile strain increases. Compressive strain, on the other hand, results in a dominant shift of the lowest conduction band at $\Delta\left(\Delta E_{C}^{\Delta}\right)$. This induces a onedimensional defect band about $300 \mathrm{meV}$ below the conduction band at moderate compressive strain $(\varepsilon \leq-0.04)$. Further increase in the dislocation-induced strain increases $\Delta E_{C}^{\Delta}$ and causes the formation of a one-dimensional defect band close to the valence band if $\varepsilon$ is below -0.08 .

Additional data about the variation of the band gap, band splitting, and effective masses with increasing strain are extracted from tight-binding simulations in combination with $\mathbf{k} \cdot \mathbf{p}$ models. Figure 6 a summarizes changes in the direct and indirect band gaps with increasing tensile or compressive strain. Besides the generally known indirect gap between the conduction-band minimum at $\Delta$ and the valence-band maximum at the $\Gamma$-point $\left(\Delta E_{\Gamma-\Delta}^{(\text {ind })}\right)$ another indirect transition with respect to the $L$-point $\left(\Delta E_{\Gamma-\Delta}^{(\text {ind })}\right)$ varies with applied strain. At $\varepsilon=+0.1$, the band gap of this transition is comparable to that of the indirect transition between $\Gamma$ and $\Delta$ (Fig. 6a). Similar data have also been published for nanowires, where, however, the absolute data depend on the wire diameter [30]. It is obvious from Fig. 6a that the direct band gap decreases monotonically

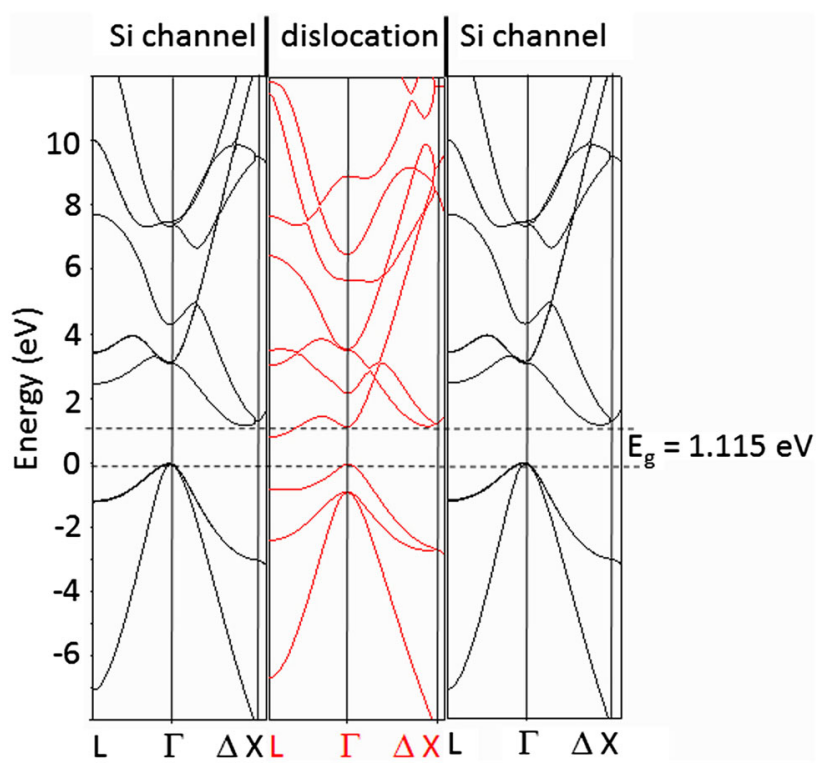

Fig. 5 Schema of the band diagram of a Si channel with a dislocation in the center. The shift of the conduction band of the strained dislocation core results in a 1-dimensional defect band in the gap of the surrounding channel. For calculation, uniaxial strain $\left(\varepsilon_{[110]}=+0.1\right.$, tensile $)$ is assumed. The valence-band maximum was set to zero 


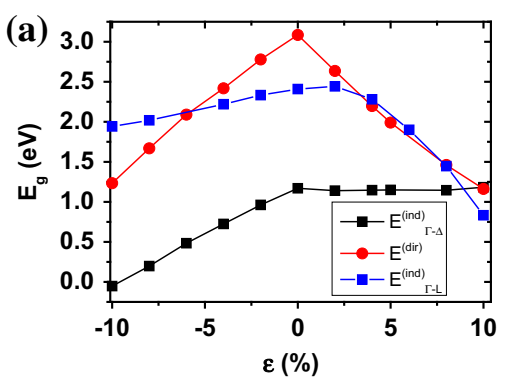

(b)

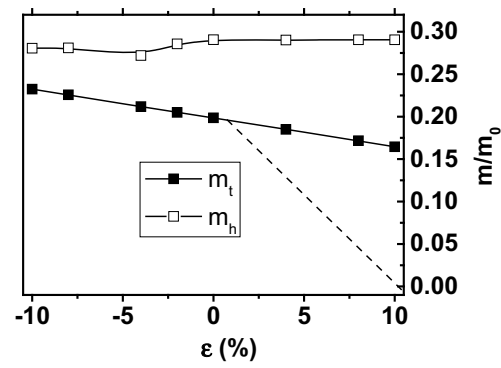

Fig. 6 Direct and indirect band gaps (a) and effective in-plane electron and hole masses (b) as a function of uniaxial strain along $<110>$ (a). Electron (full symbols) and hole masses (open symbols) are extracted from 8-band $\mathbf{k} \cdot \mathbf{p}$ data using Eq. (2). The dotted line shows the extrapolated electron mass predicted from measurements at lower tensile strain (according to Refs. [28] and [32])

with increasing uniaxial tensile or compressive strain, while the indirect band gap $\Delta E_{\Gamma-\Delta}^{(\text {ind })}$ decreases only if compressive strain is applied. The indirect band gap $\left(\Delta E_{\Gamma-\Delta}^{(\text {ind })}\right)$ is more or less constant if tensile strain exists. At a tensile strain of $10 \%(\varepsilon=+0.1)$, the direct gap is equal to the indirect gap and silicon changes over to a direct semiconductor. Furthermore, effective masses of electrons and holes have been calculated from energy dispersion curves using

$\frac{1}{m(k)}=\frac{1}{\hbar^{2}} \frac{\partial^{2} E(k)}{\partial k^{2}}$

in the close vicinity of the band extremum [31]. The calculated hole mass $m_{\mathrm{h}}=0.285$ is almost constant for $-0.1 \leq \varepsilon \leq+0.1$ and corresponds to previously published data [31]. The effective in-plane electron mass parallel to the strain direction $\left(m_{\mathrm{eff}}\right)$ decreases continuously with increasing tensile strain (Fig. 6b). The decrease in $m_{\text {eff }}$ even at higher tensile strain is much less than predicted from measurements at lower tensile strain $(\varepsilon \leq+0.01)$ [28, 32].

\section{Model of the electronic structure of dislocations}

Tensile strain induced by a dislocation shifts locally both, the valence and conduction bands causing an additional indirect transition between $\Gamma$ and $L$, as well as the direct transition at the $\Gamma$-point (Fig. 7). A cross-section through a

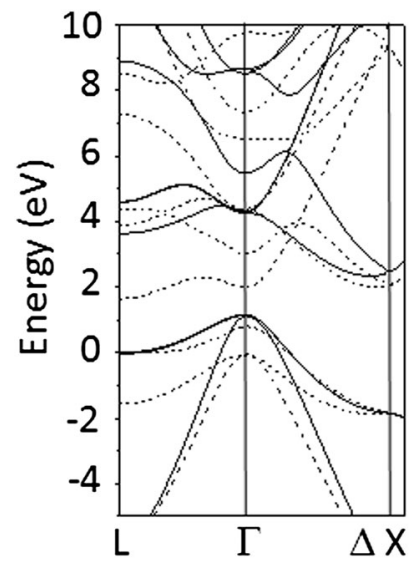

Fig. 7 Band shifts between unstrained (full lines) and strained $\mathrm{Si}$ (dashed lines). A uniaxial tensile strain of $\varepsilon_{[110]}=+0.1$ is assumed

dislocation yields a quantum well shown in Fig. 8a. Data from band structure calculations indicate the lowering of the minimum of the lowest conduction band at the $L$-point $\left(E_{\mathrm{CB}}^{L}\right)$ below that at $\Delta$ of the unstrained $\mathrm{Si}\left(E_{\mathrm{CB}}^{\Delta}\right)$. At the same time, the valence-band maximum of light and heavy holes is also shifted downwards (Fig. 7). For p-type silicon, discussed here, changes in the conduction band are most important.

Assuming the dislocation as a one-dimensional wire of radius $R$ (Fig. 4), the Schrödinger equation is written in cylindrical coordinates as $[33,34]$

$$
\begin{aligned}
& {\left[-\frac{h^{2}}{2 m_{\square}}\left(\frac{1}{r} \frac{\partial}{\partial r}\left(r \frac{\partial}{\partial r}\right)+\frac{1}{r^{2}} \frac{\partial^{2}}{\partial \Phi^{2}}+\frac{\partial^{2}}{\partial z^{2}}\right)+V_{\text {eff }}(r)\right] \varphi_{i}(r, \Phi, z)} \\
& \quad=E_{i} \varphi_{i}(r, \Phi, z) E_{i} \varphi_{i}(r, \Phi, z)
\end{aligned}
$$

with boundary conditions $\quad V_{\text {eff }}(r<R)=0 \quad$ and $V_{\text {eff }}(r>R)=\infty$. In Eq. (3), $V_{\text {eff }}$ denotes the potential, $r, \Phi$, and $z$ the cylinder coordinates as well as $\varphi_{i}(r, \Phi, z)$ the wave functions. Because only $V_{\text {eff }}$ depends on $r$, and with $\varphi_{i}(r, \Phi, z)=\varphi_{i}(r) e^{i k_{z} z}$, Eq. (3) can be rewritten as

$\left[-\frac{\hbar^{2}}{2 m_{\square}} \frac{1}{r} \frac{\partial}{\partial r}\left(r \frac{\partial}{\partial r}\right)\right] \varphi_{i}(r)=\left(E_{i}+\frac{\hbar^{2} k_{z}^{2}}{2 m_{\perp}}\right) \varphi_{i}(r)$

Solving Eq. (4) results in quantized energy levels [35]

$E_{i}=\frac{\hbar^{2}}{2 m_{\square}} \frac{J_{0}(r)^{2}}{R^{2}} \quad(n=1,2, \ldots)$

with $J_{0}(r)$ as the first root of Bessel function. With $m_{e}=0.19 m_{0}$ and $R=2.5 \mathrm{~nm}$, one obtains the two lowest energy levels $E_{1}=38.4 \mathrm{meV}$ and $E_{2}=203 \mathrm{meV}$. Both are inside the quantum wire formed by the dislocation (Fig. 8b).

The density of states (DOS) of the quantum wire was also calculated. Based on the general case [33], the onedimensional density of states is derived as 

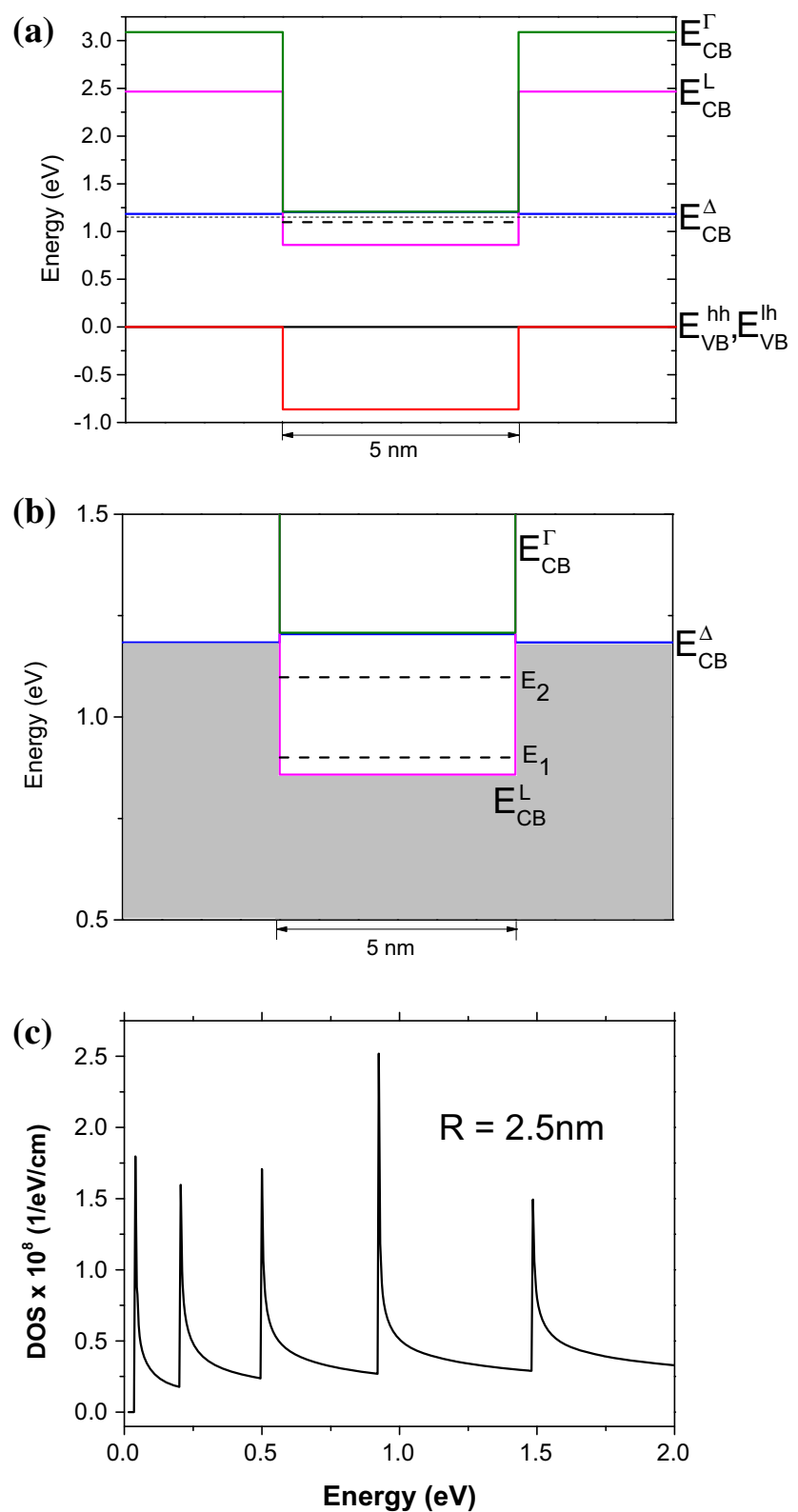

Fig. 8 Band alignment for a dislocation in a MOSFET channel schematic shown in Fig. 4. The radius of the dislocation was assumed as $R=2.5 \mathrm{~nm}$. An overview about the energies of the conductionband minima at $\Gamma, L$, and $\Delta$, as well as valence-band maxima of heavy holes (hh) and light holes (lh) extracted from band structure calculations (a). Detail of the conduction band forming the quantum wire (b). The dashed lines characterize the first energy levels calculated for the quantum wire. Density of states (DOS) calculated for a quantum wire with $R=2.5 \mathrm{~nm}(\mathbf{c})$

$$
\begin{aligned}
\operatorname{DOS}(E, \vec{r}) & =\frac{1}{\pi L^{2}} \sum_{i} \int \mathrm{d} k_{z} \delta\left(k-k_{i}\right) \frac{\delta\left(E-E_{i}\right)}{k_{z}} \\
& =\frac{1}{\pi L^{2}} \frac{m_{\perp}}{\hbar^{2}} \sum_{i} \frac{1}{\sqrt{\frac{2 m_{\perp}}{\hbar^{2}}\left(E-E_{i}\right)}}
\end{aligned}
$$

$\operatorname{DOS}(E) L^{2}=\frac{1}{\pi} \frac{\sqrt{2 m_{\perp}}}{\hbar} \sum_{i} \frac{1}{\sqrt{E-E_{i}}}$

where $L=2 R$ and $E_{i}$ denotes the energy levels given by Eq. (5). Using $R=2.5 \mathrm{~nm}$ and $E_{1}-E_{5}$, the density of states for the quantum wire is plotted in Fig. 8c.

The interpretation of dislocations as quantum wires explains most of the experimental results. The presence of $\mathrm{SdH}$ oscillations refers to a $2 \mathrm{DEG}$ if two-dimensional dislocation networks exist in the channel of MOSFETs (Fig. 2). Because a network is formed by periodically spaced dislocations, the $2 \mathrm{DEG}$ represents a two-dimensional set of one-dimensional electron gases (1DEG). Moreover, the energy quantization is related to the presence of Coulomb blockades proved by low-temperature measurements. The model of dislocations as quantum wires bears similarities to the previously published shallow band model, which, however, assumes 1D-bands about 50-100 meV below the conduction and above the valence band, respectively [8, 36, 37]. Deeper defect-induced states of minority or majority carriers proved by DLTS $[6,11,38]$ are not considered therein, but are explainable on the basis of the quantum wire model. If atomic defects on the dislocation core are taken into account (kinks, jogs, etc. [25]) then local changes in strain along the dislocation line exist modifying locally the band structure and consequently the quantum wire. Therefore, a broad defect line appears in the DLTS spectrum.

An analogous discussion of the formation of quantum wells by dislocation-induced compressive strain appears also possible which, however, is based on the primary indirect transition between $\Gamma$ and $\Delta$.

\section{Conclusions}

Detrimental effects on electronic parameters have been stated for dislocations if they are randomly placed in active areas of semiconductor devices. Presented experimental data, however, show a significant increase in device performance (especially of the drain current), if defined numbers and types of pure dislocations are placed in the channel of MOSFETs and directly connected to source and drain. The drain current enhancement is induced by exceptional electronic properties of dislocations, namely by supermetallic conductivity.

The presented model of the electronic properties of dislocations is based on the high strain in the defect core. It is shown that the high strain level on the dislocation core in the order of $10 \%$ or more modifies the electronic band structure resulting in the formation of a quantum wire inside the silicon channel. The lateral size of the quantum 
wire is effected by the core diameter of the dislocation and is in the order of a few nanometers. The formation of the quantum wire forces carrier confinement along the dislocation line and generates a 1DEG. Most important for the exceptional properties is the energy quantization instead of the effective mass. Assuming, for instance, a wire radius $R=2.5 \mathrm{~nm}$, the lowest energy level is $E_{1}=38.4 \mathrm{meV}$ (Eq. 5) which corresponds to experimentally measured distances of Coulomb blockades [20].

The utilization of the exceptional electronic properties of dislocations enables new concepts in development of future device generations. The main advantages in integration of defined numbers and types of dislocations are (1) further application of silicon-based CMOS processes below $10 \mathrm{~nm}$ feature size, (2) easy production processes of dislocation arrangements also in very thin SOI layers, (3) their thermal stability over process steps, and (4) the use of native nanostructures without improved lithography and additional non-silicon materials.

Open Access This article is distributed under the terms of the Creative Commons Attribution 4.0 International License (http://crea tivecommons.org/licenses/by/4.0/), which permits unrestricted use, distribution, and reproduction in any medium, provided you give appropriate credit to the original author(s) and the source, provide a link to the Creative Commons license, and indicate if changes were made.

\section{References}

1. C. Kittel, Introduction to Solid State Physics (Hoboken, NJ, 2005)

2. D. Hull, D.J. Bacon, Introduction to Dislocations (Pergamon, Oxford, 1994)

3. D.B. Holt, B.G. Yacobi, Extended Defects in Semiconductors (Cambridge University Press, Cambridge, 2007)

4. R. Labusch, W. Schröter, in Dislocations in Solids, vol. 5, ed. by F.R.N. Nabarro (North-Holland, Amsterdam, 1980), p. 127

5. H. Alexander, H. Teichler, in Materials Science and Technology, vol. 4, ed. by R.W. Cahn, P. Haasen, E.J. Kramer (VCH, Weinheim, 1991), p. 249

6. W. Schröter, H. Cerva, Solid State Phenom. 85-86, 67 (2002)

7. V. Kveder, M. Kittler, W. Schröter, Phys. Rev. B 63, 115208 (2001)

8. V. Kveder, M. Kittler, Mat. Sci. Forum 590, 29 (2008)

9. M. Trushin, O.F. Vyvenko, T. Mchedlidze, O. Kononchuk, M. Kittler, Solid State Phenom. 156-158, 282 (2010)

10. I. Isakov, A. Bondarenko, O.F. Vyvenko, V. Vdovin, E. Ubyivovk, O. Kononchuk, J. Phys. Conf. Ser. 281, 012010 (2011)

11. A. Bondarenko, O.F. Vyvenko, I. Isakov, Semiconductors 47, 223 (2013)
12. M. Reiche, M. Kittler, H. Uebensee, E. Pippel, S. Hopfe, Adv. Nano Res. 2, 1 (2014)

13. M. Reiche, M. Kittler, W. Erfurth, E. Pippel, K. Sklarek, H. Blumtritt, A. Haehnel, H. Uebensee, J. Appl. Phys. 115, 194303 (2014)

14. M. Reiche, M. Kittler, D. Buca, A. Hähnel, Q.-T. Zhao, S. Mantl, U. Gösele, Jpn. J. Appl. Phys. 49, 04DJ02 (2010)

15. S. Birner, T. Zibold, T. Andlauer, T. Kubis, M. Sabathil, A. Trellakis, P. Vogl, I.E.E.E. Trans, Electron. Dev. 54, 2137 (2007)

16. J.-M. Jancu, R. Scholz, F. Beltram, F. Bassani, Phys. Rev. B 57, 6493 (1998)

17. R.J. Haug, K. von Klitzing, K. Ploog, Phys. Rev. B 35, 5933 (1987)

18. N. Griffin, R.B. Dunford, M. Pepper, D.J. Robbins, A.C. Churchill, W.Y. Leong, J. Phys. Condens. Matter 12, 1811 (2000)

19. W. Shim, J. Ham, J. Kim, W. Lee, Appl. Phys. Lett. 95, 232107 (2009)

20. M. Reiche, M. Kittler, M. Schmelz, R. Stolz, H. Uebensee, M. Kehrmann, T. Ortlepp, J. Phys Conf. Ser. 568, 052024 (2014)

21. P.L. Galindo, S. Kret, A.M. Sanchez, J.-Y. Laval, A. Yanez, J. Pizarro, E. Guerrero, T. Ben, S.I. Molina, Ultramicroscopy 107, 1186 (2007)

22. H. Alexander, H. Gottschalk, C. Kiselowski-Kemmerich, in Dislocations in Solids, ed. by H. Suzuki, T. Ninomiya, K. Sumino, S. Takeuchi (University of Tokyo Press, Tokyo, 1985), p. 337

23. A. T. Blumenau, $\mathrm{PhD}$ thesis, University of Paderborn, 2002

24. C.W. Zhao, Y.M. Xing, Proc. of SPIE 7375, 737508 (2009)

25. J.P. Hirth, J. Lothe, Theory of dislocations (Wiley, New York, 1982)

26. M.V. Fischetti, Z. Ren, P.M. Solomon, M. Yang, K. Rim, J. Appl. Phys. 94, 1079 (2003)

27. V. Sverdlov, Strain-induced effects in advanced MOSFETs (Springer, Wien, 2011)

28. T. Maegawa, T. Yamauchi, T. Hara, H. Tsuchiya, M. Ogawa, I.E.E.E. Trans, Electron. Dev. 56, 553 (2009)

29. E. Ungersboeck, S. Dhar, G. Karlowatz, V. Sverdlov, H. Kosina, S. Selberherr, I.E.E.E. Trans, Electron. Dev. 54, 2183 (2007)

30. D. Shiri, Y. Kong, A. Buin, M.P. Anantram, Appl. Phys. Lett. 93, 073114 (2008)

31. S.Z. Karazhanov, A. Davletova, A. Ulyashin, J. Appl. Phys. 104, $024501(2008)$

32. E. Ungersboeck, S. Dhar, G. Karlowatz, H. Kosina, S. Selberherr, J. Comput. Electron. 6, 55 (2007)

33. J.M. Ziman, Principles of the Theory of Solids (Cambridge University Press, Cambridge, 1998)

34. H. Reinhardt, Quantenmechanik 1: Pfadintegralformulierung und Operatorformalismus (Oldenbourg Verlag, Munich, 2012)

35. W. Lu, J. Xiang, B.P. Timko, Y. Wu, C.M. Lieber, PNAS 102, 10046 (2005)

36. M. Kittler, M. Reiche, M. Krause, Solid State Phenom. 205-206, 293 (2014)

37. M. Reiche, M. Kittler, M. Krause, H. Übensee, A.I.P. Conf, Proc. 1583, 33 (2014)

38. K. Knobloch, W. Seifert, M. Kittler, Mater. Sci. Eng., B 42, 254 (1996) 